\title{
Myasthenia gravis exacerbation and myasthenic crisis associated with COVID-19: case series and literature review
}

\author{
Cleonisio Leite Rodrigues ${ }^{1} \cdot$ Hermany Capistrano de Freitas ${ }^{1} \cdot$ Paulo Reges Oliveira Lima ${ }^{2}$. \\ Pedro Helder de Oliveira Junior ${ }^{2}$. José Marcelino Aragão Fernandes ${ }^{2}$. José Artur Costa D’Almeida ${ }^{1}$. \\ Paulo Ribeiro Nóbrega ${ }^{2}$ (1)
}

Received: 28 August 2021 / Accepted: 10 December 2021 / Published online: 18 January 2022

(c) Fondazione Società Italiana di Neurologia 2021

\begin{abstract}
Background Myasthenia gravis (MG) is an autoimmune disorder of the neuromuscular junction that can be exacerbated by many viral infections, including COVID-19. The management of MG exacerbations is challenging in this scenario. We report 8 cases of MG exacerbation or myasthenic crisis associated with COVID-19 and discuss prognosis and treatment based on a literature review.

Results Most patients were female (7/8), with an average age of 47.1 years. Treatment was immunoglobulin (IVIG) in 3 patients, plasma exchange (PLEX) in 2 patients, and adjustment of baseline drugs in 3. In-hospital mortality was $25 \%$ and $37.5 \%$ in 2-month follow-up.

Discussion This is the largest case series of MG exacerbation or myasthenic crisis due to COVID-19 to this date. Mortality was considerably higher than in myasthenic crisis of other etiologies. Previous treatment for MG or acute exacerbation treatment did not seem to interfere with prognosis, although sample size was too small to draw definitive conclusions. Further studies are needed to understand the safety and effectiveness of interventions in this setting, particularly of PLEX, IVIG, rituximab, and tocilizumab.
\end{abstract}

Keywords Myasthenia gravis · Neuromuscular junction diseases $\cdot$ Myasthenic crisis · Neurological symptoms ·

Coronavirus $\cdot$ COVID-19

\section{Introduction}

In mid-November 2019, an outbreak of pneumonia of unknown cause in the city of Wuhan, Hubei province, China, began to attract the attention of Chinese health authorities [1]. The virus was identified as a new type of coronavirus $(\mathrm{CoV})$, named SARS-CoV-2, and its associated disease received the name of COVID-19, which was declared a pandemic by the World Health Organization Health on March 11, 2020, with high levels of contamination and mortality in China, Italy, and Spain and later in the USA and Brazil [1].

Paulo Ribeiro Nóbrega

paulo_r_med@yahoo.com.br

1 Department of Neurology, Hospital Geral de Fortaleza, Fortaleza, Brazil

2 Division of Neurology, Department of Clinical Medicine, Universidade Federal Do Ceará, Fortaleza, Brazil
Myasthenia gravis (MG) is an autoimmune disorder that affects the neuromuscular junction and may lead to respiratory muscle weakness with respiratory insufficiency (myasthenic crisis) [2]. It can often be exacerbated by common viral or bacterial infections, including COVID-19 infection [3].

Exacerbation of MG during COVID-19 infection appears to be uncommon, ranging from 10 to $15 \%[4,5]$. The scenario of myasthenia exacerbation due to COVID-19 can be challenging, both due to diagnostic concerns (is respiratory insufficiency related to hypoventilation secondary to muscle weakness or hypoxia secondary to pulmonary involvement?) and treatment (are plasma exchange [PLEX] and intravenous immunoglobulin [IVIG] safe and effective for the treatment of myasthenic crisis in this setting?).

Previous studies have reported outcomes of myasthenic patients with COVID-19 infection, but few of them have focused especially on those who had exacerbations. We report 8 cases of myasthenia exacerbation or myasthenic crisis 
associated with acute COVID-19 infection and discuss prognosis and treatment based on a literature review.

\section{Material and methods}

This was an observational retrospective study including all consecutive adult patients diagnosed with MG (based on antibodies or on electrophysiology) and admitted with acute COVID-19 infection and myasthenia exacerbation at two reference hospitals in Fortaleza, state of Ceará in the northeast of Brazil, from May 2020 to April 2021. Diagnosis of COVID-19 was confirmed with real-time reversetranscriptase polymerase-chain-reaction (RT-PCR) assay (LightMIx Modular SARS-CoV (COVID-19) Egene and LightMIx Modular SARS-CoV (COVID-19) RdRP, Roche Diagnostics S.L.) from oropharyngeal-nasopharyngeal swab, sputum, or lower respiratory tract in all cases. Informed consent was waived because of the retrospective observational nature of the study and the analysis used anonymous clinical data.

All patients underwent detailed clinical and neurological examination, and neuromuscular specialists in each hospital reviewed medical charts. The diagnosis of MG is based on clinical aspects, serology (positive acetylcholine receptorAchR autoantibodies, or muscle-specific tyrosine kinase (MuSK) antibodies), and electrophysiological findings (abnormal decrement in repetitive nerve stimulation-RNS). Myasthenia Gravis Foundation of America (MGFA) scores [6] were defined based on the clinical descriptions 1 month prior to hospitalizations when available. MG exacerbation was defined as a MGFA score worsening from baseline or respiratory insufficiency needing mechanical ventilation (MV).

\section{Results}

We identified 8 patients with myasthenia exacerbation or myasthenic crisis during acute COVID-19 infection (Table 1). Most patients were female (7/8), with an average age of 47.1 years. The majority (6/8) required mechanical ventilation.

All patients had MGFA class increase denoting MG exacerbation. Most patients (6/8) had worsening of previously stable MG, while 2 patients presented initial MG symptoms during COVID-19 infection. Diagnostic confirmation was done with RNS in 7 patients and antibodies in 7 (6 with AChR and 1 with MuSK antibodies). Of the patients with an initial diagnosis of MG, one had anti-AChR antibodies, and the other had negative AChR and MUSK antibodies but marked decremental response on RNS.
Treatment of exacerbation was IVIG in 3 patients, PLEX in 2 patients, and adjustment of baseline myasthenia drugs in 3. All patients who received IVIG survived, and one of the two patients who received PLEX died. Average hospital stay was 42.62 days. Mortality during hospital stay was $2 / 8$ and $3 / 8$ in short-term follow-up ( 2 months). Of those with myasthenic crisis, mortality in follow-up was 50\% (3/6).

Antibiotics were prescribed to 7 patients, including classes of antibiotics reported to worsen MG. Half of the patients (4/8) also received hydroxychloroquine, azithromycin, or ivermectin.

We present the details, clinical course, and treatment of two illustrative cases that exemplify the diagnostic and therapeutic challenges in these patients.

\section{Case 1}

A 37-year-old female patient started with dysphagia, dysphonia, and post-prandial cough, followed by bilateral ptosis, diplopia, and mild dyspnea on exertion 3 months before admission. There was no previous illness and no family history of neurologic diseases. Three months later, she had sudden worsening of dyspnea, needing supplementary oxygen, and was intubated the following day. Arterial blood gas analysis revealed hypercapnic respiratory failure. RTPCR was positive for SARS-Cov-2 infection. Chest highresolution CT scan revealed a pattern suggestive of COVID19 pneumonia. Five days later, after waning of sedation, bilateral ptosis, facial weakness, and ophthalmoparesis were perceived, and a clinical diagnosis of MG was suggested (characterized as MGFA class IIIB based on previous symptoms). She received dexamethasone treatment for COVID-19 pneumonia and was submitted to PLEX. Anti-AChR and MuSK antibodies were negative, but a marked decremental response on RNS was present. She underwent tracheostomy and was successfully weaned from mechanical ventilation. After 1 month of admission, she had another exacerbation with worsening of ptosis, dyplopia, and dysphagia and underwent another five sessions of PLEX. Considering her MG to be refractory, rituximab $750 \mathrm{mg} / \mathrm{m}^{2}$ in two divided doses was administered. After 1 week of initial rituximab infusion, she had significant improvement, was decannulated, and discharged home on prednisone $60 \mathrm{mg} / \mathrm{day}$ and pyridostigmine.

\section{Case 2}

A 49-year-old female patient had been diagnosed with generalized MG since 2013 (clinical findings, abnormal RNS, and positive AchR autoantibodies), MGFA class IIa, having been treated with azathioprine, prednisone, and pyridostigmine. In 2015, she was diagnosed with thymoma and underwent thymectomy and radiotherapy. Two days before 


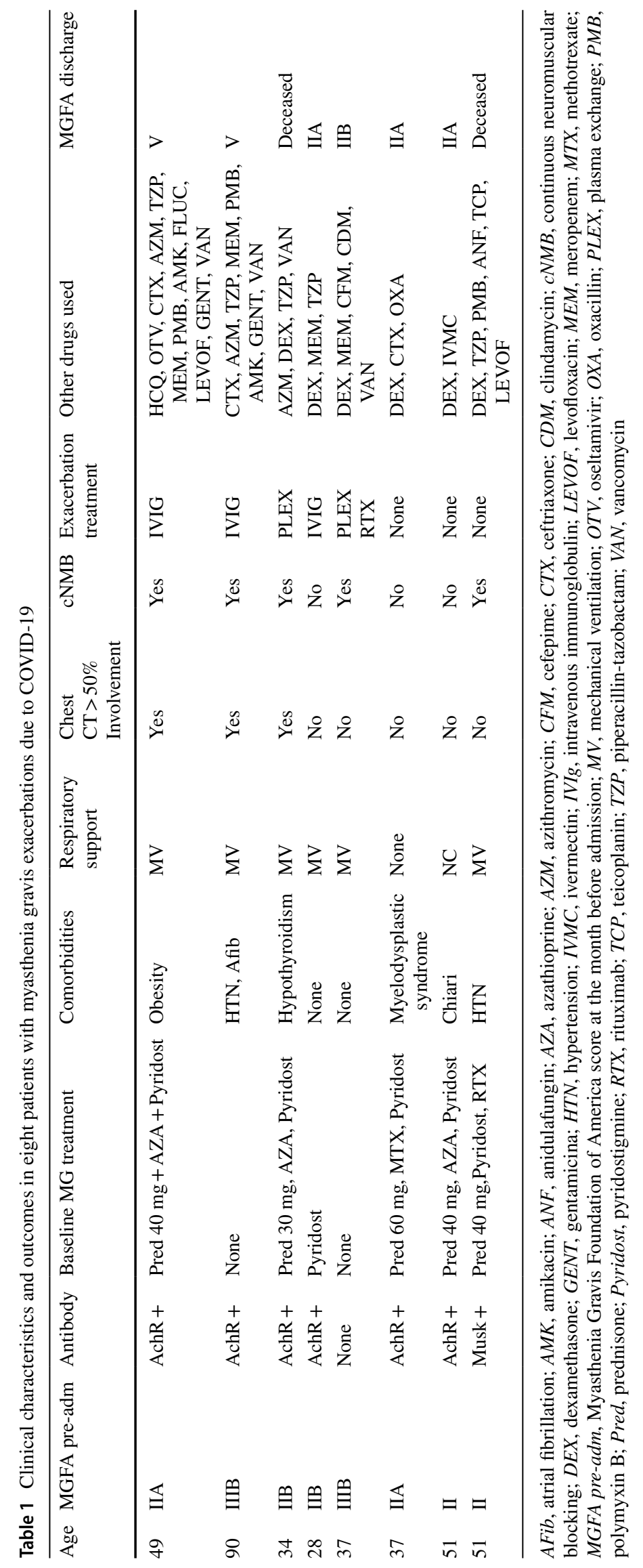


admission in May 2020, the patient reported fever, shivering, odynophagia, headache, and dyspnea. One day before admission, she also reported cough. RT-PCR was positive for SARS-Cov-2 infection. After admission, dyspnea got worse; ptosis and muscle weakness in four limbs were noted. Chest high-resolution CT scan revealed a pattern suggestive of COVID-19 pneumonia. She was transferred to an intensive care unit. Azathioprine was suspended due to suspected associated bacterial infection; prednisone dosage was increased to $60 \mathrm{mg} /$ day. Five days after admission, patient had hypercapnic respiratory failure and required orotracheal intubation, remaining for 30 days under sedation and neuromuscular block. During this period, she performed 2 cycles (5 days each) of IVIG at a dose of $1 \mathrm{~g} / \mathrm{kg}$ at an interval of 21 days. After extensive treatment with antibiotics for bacterial pneumonia and sepsis, she achieved clinical improvement but still needed prolonged mechanical ventilation through a tracheostomy. She was discharged 2 months after admission still using a tracheostome but without the need for mechanical ventilation. She is currently decannulated, independent for activities of daily living, and returned to her previous treatment with azathioprine $150 \mathrm{mg}$ /day, pyridostigmine, and prednisone $20 \mathrm{mg} /$ day.

\section{Discussion}

This is one of the first case series to include only patients with myasthenia exacerbation or myasthenic crisis triggered by acute COVID-19 infection. We discuss clinical characteristics, pulmonary and systemic involvement, previous MG treatment, and acute exacerbation treatment and prognosis in our patients and compare to a literature review.

Viral and bacterial respiratory infections are common triggers for exacerbation in MG. COVID-19 has also been described as a trigger for exacerbation [3, 7-9], although MG worsening appears to be uncommon in COVID, affecting only $10-15 \%$ of patients $[4,5]$. Comparing this number to seasonal influenza, a recent study performed before the coronavirus pandemic has shown that an influenza-like illness resulted in exacerbation in $40 \%$ of myasthenic patients [10]. In the same study, a common cold resulted in exacerbation in $15.6 \%$. It is still unclear if this discrepancy is a result of underreporting of MG worsening due to severity of pulmonary symptoms in COVID-19 or if it represents a lower potential for exacerbating MG symptoms in COVID-19 infection.

Some studies have established MG as an independent risk factor for worse prognosis in COVID-19 [4] and found that unsatisfactory control of MG symptoms, long-term corticosteroid treatment, older age, cancer, and rituximab use were risk factors for death in these patients. However, very few of these patients had MG exacerbations, and death was related to pulmonary involvement by viral or secondary pneumonia in most cases. In the present study, we systematically focus on patients with exacerbations.

The impact of plasma exchange in COVID-19 has been under study recently. One of the main causes of tissue damage in COVID-19 is a widespread immune dysregulation referred to as cytokine storm syndrome (CSS) with elevated interleukin-1 (IL-1), interleukin-6 (IL-6), granulocyte colony-stimulating factor (GCSF), tumor necrosis factor (TNF), ferritin, and other immune-inflammatory mediators [11]. Some of these factors could be removed by plasma exchange. In a case series of 31 patients with acute respiratory distress syndrome (ARDS) due to SARS-CoV-2, PLEX has been shown to reduce mortality and improve extubation rates [12]. PLEX has also reduced mortality in secondary thrombotic microangiopathy (TMA) and diffuse intravascular coagulation (DIC) [13, 14]. Major concerns involving PLEX are hypotension, which can be partially overcome with administration of intravenous fluids [15], and risk of bleeding due to procoagulant depletion. Plasma exchange also leads to immunosuppression. Immediately after PLEX the levels of IgA, IgG, IgM, C3, C4, and CH50 are reduced by $50-70 \%$ [16]. Some authors have found high infection rates in patients undergoing PLEX [17], but we found no robust evidence based on controlled trials to quantify that risk. In our series, two patients were treated with PLEX. One of them died, but the other had a very significant improvement and was discharged in a better MGFA class than before COVID-19 infection.

Intravenous immunoglobulin (IVIG) has anti-inflammatory and immunomodulating effects. Previous studies have shown efficacy of IVIG in the treatment of patients with severe inflammatory response associated with influenza [18] and SARS-CoV [19] infection, but there are not sufficient evidence to ascertain its safety and effectiveness in COVID19. There are reports of myasthenic exacerbations due to COVID-19 treated with IVIG without major side effects [20]. Two major side effects are thrombotic events that have an estimated incidence of 1-16.9\% [21] and transfusionrelated acute lung injury (TRALI), an immune-mediated process that can lead to ARDS [21]. Three patients in our series received IVIG. None of them died. One was discharged in a better MGFA than at admission and the other two in a worse functional status.

Medications used for chronic immunosuppressive treatment in MG might also influence COVID-19 disease course. Evidence from small series suggested that corticosteroids in higher doses and rituximab might increase mortality. Azathioprine, mycophenolate mofetil, and cyclosporine did not influence outcomes [4]. Of the 3 patients in our series who died during follow-up, two were taking corticosteroids, one of them associated with azathioprine and the other with rituximab. The third patient was not using any medication as she did not have a previous diagnosis of MG before 
myasthenic crisis. Among survivors, most patients also used corticosteroids chronically (3/5). Only two out of 8 patients did not need mechanical ventilation, and both were taking oral prednisone $>40 \mathrm{mg} / \mathrm{kg}$. Both these patients had a very short hospital state and were discharged in a similar MGFA to the one they were in before admission.

Some drugs which have been used for general supportive care in COVID-19 might also exacerbate myasthenic symptoms. Three of our patients took azithromycin, a macrolide antibiotic that may be able to suppress inflammation in ARDS [22] but has failed to demonstrate clinical efficacy in COVID-19 [23]. Two of these patients died (66\%). Azithromycin is known to worsen myasthenic symptoms and may precipitate myasthenic crisis [24, 25]. All patients taking azithromycin required mechanical ventilation. Only one patient received hydroxychloroquine, another drug that has shown in vitro antiviral activity but has failed to demonstrate clinical benefit [26]. Chloroquine and hydroxychloroquine also have the potential to exacerbate MG symptoms [24, 25].

Antibiotics may be prescribed in critically ill COVID19 patients to treat known or suspected secondary bacterial infection. Many antibiotics may also exacerbate MG. In our series, some patients received aminoglycosides (gentamicin and amikacin), clindamycin, and polymyxin, which are all on the list of medications to be avoided in myasthenic patients. The small sample and absence of a control group prevent us from assessing possible effects of these drugs on clinical course. Care should be taken when treating any myasthenic patient with antibiotics.

Our case series showed a very high mortality rate of $37.5 \%$ (3/8) on short-term follow-up. Two of these patients died during hospitalization, and another died 2 months after discharge. Cause of death was septic shock in two patients and unknown in the one who died at home. A study with COVID-19 patients requiring hospitalization in Brazil revealed ICU admission rates of $39 \%$ and in-hospital mortality rate of 38\% [27]. Mortality in myastenic crisis of varied etiologies was $12 \%$ in a large case series, and main cause of death was multiorgan failure, mostly due to sepsis [28]. Of the patients who had myasthenic crisis in our series, mortality was even higher $(3 / 6,50 \%)$. Despite our small sample, it is possible that mortality of myasthenic crisis due to COVID-19 is considerably higher than that of MG crisis due to other etiologies.

Considering current evidence, we believe that the decision to treat MG exacerbations in COVID-19 with PLEX or immunoglobulin should be taken based on particularities of individual patients, such as risk of thrombotic complications, secondary bacterial or fungal infections, and hemodynamic parameters. Immunoglobulin probably results in less hemodynamic compromise and less interference with cytokines, chemokines, and innate immunity factors (which might have a positive or negative effect on outcomes) but might increase the risk of thrombosis, which is also high in COVID-19 infections. PLEX has been studied for the treatment of cytokine storm syndrome in COVID, although evidence is limited to small studies and potential risks such as bleeding, hypotension, and possible predisposition to secondary infections raise concerns about its widespread use.

The main limitation of our study is that it was an observational retrospective study with a small number of patients, which limited comparative statistical analysis. Nevertheless, this is the largest case series to date to include only patients with MG exacerbation and myasthenic crisis due to COVID-19.

\section{Conclusion}

Myasthenia exacerbations and myasthenic crisis seem to be uncommon complications of COVID-19 infections. Prognosis in these patients, however, might be very poor. Further studies are needed to understand the safety and effectiveness of interventions in this setting, particularly of PLEX, IVIG, rituximab, and tocilizumab.

Acknowledgements We would like to acknowledge the staff in both participating centers for their hard work and support.

Author contribution The authors confirm contribution to the paper as follows: study conception and design, Paulo Ribeiro Nóbrega and Cleonísio Leite Rodrigues; data collection, Hermany Capistrano Freitas, Paulo Reges Oliveira Lima, Pedro Helder de Oliveira Junior, Paulo Ribeiro Nóbrega, and José Marcelino Aragão Fernandes; analysis and interpretation of results, Paulo Ribeiro Nóbrega, Cleonísio Leite Rodrigues, and José Artur Costa D 'Almeida; and manuscript draft preparation, Paulo Ribeiro Nóbrega, Cleonísio Leite Rodrigues, and Hermany Capistrano Freitas. All authors reviewed the results and approved the final version of the manuscript.

Funding The research was funded by the researchers.

Data availability All materials used in this study will be made available subject to a material transfer agreement.

Code availability Not applicable.

\section{Declarations}

Ethical approval The study was approved by the local ethics committee under the number 4881736.

Consent to participate Informed consent was waived because of the retrospective observational nature of the study and the analysis used anonymous clinical data as approved by the research ethics committee.

Consent for publication The manuscript contains no individual person's data in any form.

Conflict of interest The authors declare no competing interests. 


\section{References}

1. World Health Organization. Coronavirus disease (COVID-19) pandemic. Published online 2021. https://www.who.int/emergencies/ diseases/novel-coronavirus-2019. Accessed 16 June 2021

2. Bedlack RS, Sanders DB (2002) On the concept of myasthenic crisis. J ClinNeuromusculDis 4(1):40-42. https://doi.org/10.1097/ 00131402-200209000-00009

3. Camelo-Filho AE, Silva AMS, Estephan EP, et al (2020) Myasthenia gravis and COVID-19: clinical characteristics and outcomes. Front Neurol 11(September). https://doi.org/10.3389/fneur.2020.01053

4. Jakubíková M, Týblová M, Tesař A et al (2021) Predictive factors for a severe course of COVID-19 infection in myasthenia gravis patients with an overall impact on myasthenic outcome status and survival. Eur J Neurol 870177:1-8. https://doi.org/10.1111/ene.14951

5. Businaro P, Vaghi G, Marchioni E, et al (2021) COVID-19 in patients with myasthenia gravis: epidemiology and disease course. Muscle Nerve (May):1-6.https://doi.org/10.1002/mus.27324

6. Jaretzki A, Barohn RJ, Ernstoff RM et al (2000) Myasthenia gravis: recommendations for clinical research standards. Neurology 55(1):16-23. https://doi.org/10.1212/WNL.55.1.16

7. Rein N, Haham N, Orenbuch-Harroch E et al (2020) Description of 3 patients with myasthenia gravis and COVID-19. J Neurol Sci 417:117053. https://doi.org/10.1016/j.jns.2020.117053

8. Anand P, Slama MCC, Kaku M et al (2020) COVID-19 in patients with myasthenia gravis. Muscle Nerve 62(2):254-258. https://doi. org/10.1002/mus.26918

9. Županić S, PerićŠitum M, Majdak M, Karakaš M, Bašić S, Sporiš D Case series of COVID-19 in patients with myasthenia gravis: a single institution experience. Acta Neurol Belg Published online 2021. https://doi.org/10.1007/s13760-021-01662-w

10. Seok HY, Shin HY, Kim JK, Kim BJ, Oh J, Bum C, Sun YK, Kang SY, Ahn S-W, Bae JS, Kim B-J (2017) The impacts of influenza infection and vaccination. J Clin Neuro 13(4):325-330

11. Yuki K, Fujiogi M, Koutsogiannaki S 2020 COVID-19 pathophysiology: a review. Clin Immunol 215. https://doi.org/10.1016/j.clim. 2020.108427

12. Khamis F, Al-Zakwani I, Al Hashmi S, Al Dowaiki S, Al Bahrani M, Pandak N, Al Khalili H, Memish Z. 2020 Therapeutic plasma exchange in adults with severe COVID-19 infection. Int $\mathrm{J}$ Infect Dis 99:214-218. https://doi.org/10.1016/j.ijid.2020.06.064

13. Pene F, Vigneau C, Auburtin M et al (2005) Outcome of severe adult thrombotic microangiopathies in the intensive care unit. Intensive Care Med 31(1):71-78. https://doi.org/10.1007/s00134-004-2505-0

14. Chang JC (2019) Acute respiratory distress syndrome as an organ phenotype of vascular microthrombotic disease: based on hemostatic theory and endothelial molecular pathogenesis. Clin Appl Thromb 25. https://doi.org/10.1177/1076029619887437

15. Sarabjot SM (2020) Therapeutic plasma exchange and COVID-19: a rapid review. Clin Immunol Immunother 6(4):1-5. https://doi.org/ 10.24966/ciit-8844/1000041

16. Keller AJ, Urbaniak SJ (1978) Intensive plasma exchange on the cell separator: effects on serum immunoglobulins and complement components. Br J Haematol 38(4):531-540. https://doi.org/10.1111/j. 1365-2141.1978.tb01078.x

17. Wing EJ, Bruns FJ, Fraley DS, Segel DP, Adler S (1980) Infectious complications with plasmapheresis in rapidly progressive glomerulonephritis. JAMA J Am Med Assoc 244(21):2423-2426. https:// doi.org/10.1001/jama.1980.03310210025020

18. Liu Q, Zhou YH, Yang ZQ (2016) The cytokine storm of severe influenza and development of immunomodulatory therapy. Cell Mol Immunol 13(1):3-10. https://doi.org/10.1038/cmi.2015.74
19. Hung IFN, To KKW, Lee CK et al (2013) Hyperimmune IV immunoglobulin treatment: a multicenter double-blind randomized controlled trial for patients with severe 2009 influenza A(H1N1) infection. Chest 144(2):464-473. https://doi.org/10.1378/chest.12-2907

20. Costamagna G, Abati E, Bresolin N, Comi GP, Corti S (2021) Management of patients with neuromuscular disorders at the time of the SARS-CoV-2 pandemic. J Neurol 268(5):1580-1591. https://doi. org/10.1007/s00415-020-10149-2

21. Woodruff RK, Grigg AP, Firkin FC, Smith IL (1986) Fatal thrombotic events during treatment of autoimmune thrombocytopenia with intravenous immunoglobulin in elderly patients. Lancet 328(8500):217-218. https://doi.org/10.1016/s0140-6736(86) 92511-0

22. Haydar D, Cory TJ, Birket SE et al (2019) Azithromycin polarizes macrophages to an M2 phenotype via inhibition of the STAT1 and NF-KB signaling pathways. J Immunol 203(4):1021-1030. https:// doi.org/10.4049/jimmunol.1801228

23. Rosenberg ES, Dufort EM, Udo T, Wilberschied LA, Kumar J, Tesoriero J, Weinberg P, Kirkwood J, Muse A, DeHovitz J, Blog DS, Hutton B, Holtgrave DR, Zucker HA (2020) Association of treatment with hydroxychloroquine or azithromycin with in-hospital mortality in patients with COVID-19 in New York State. JAMA 323(24):2493-2502. https://doi.org/10.1001/jama.2020.8630

24. Van Berkel MA, Twilla JD, England BS (2016) Emergency department management of a myasthenia gravis patient with communityacquired pneumonia: does initial antibiotic choice lead to cure or crisis? J Emerg Med 50(2):281-285. https://doi.org/10.1016/j.jemer med.2015.04.019

25. Sheikh S, Alvi U, Soliven B, Rezania K (2021) Drugs that induce or cause deterioration of myasthenia gravis: an update. J Clin Med 10(7):1537. https://doi.org/10.3390/jcm10071537

26. AB Cavalcanti, FG Zampieri, RG Rosa, LCP Azevedo, VC Veiga, A Avezum, LP Damiani, A Marcadenti, L Kawano-Dourado, T Lisboa, DLM Junqueira, E de Barros PGM Silva, L Tramujas, EO AbreuSilva, LN Laranjeira, AT Soares, LS Echenique, AJ Pereira, FGR Freitas, OCE Gebara, VCS Dantas, RHM Furtado, EP Milan, NA Golin, FF Cardoso, IS Maia, CR Hoffmann Filho, APM Kormann, RB Amazonas, MF Bocchi de Oliveira, A Serpa-Neto, M Falavigna, RD Lopes, FR Machado, Berwanger O; Coalition Covid-19 Brazil I Investigators (2020) Hydroxychloroquine with or without azithromycin in mild-to-moderate COVID-19. N Engl J Med 383(21):2041-2052. https://doi.org/10.1056/NEJMoa2019014

27. Ranzani OT, Bastos LSL, Gelli JGM, Marchesi JF, Baião F, Hamacher S, Bozza FA (2021) Characterisation of the first 250,000 hospital admissions for COVID-19 in Brazil: a retrospective analysis of nationwide data. Lancet Respir Med 9(4):407-418. https://doi. org/10.1016/S2213-2600(20)30560-9

28. Neumann B, Angstwurm K, Mergenthaler P, Kohler S, Schönenberger S, Bösel J, Neumann U, Vidal A, Huttner HB, Gerner ST, Thieme A, Steinbrecher A, Dunkel J, Roth C, Schneider H, Schimmel E, Fuhrer H, Fahrendorf C, Alberty A, Zinke J, Meisel A, Dohmen C, Stetefeld HR; German Myasthenic Crisis Study Group (2020) Myasthenic crisis demanding mechanical ventilation: a multicenter analysis of 250 cases. Neurology 94(3):e299-e313. https:// doi.org/10.1212/WNL.0000000000008688

Publisher's note Springer Nature remains neutral with regard to jurisdictional claims in published maps and institutional affiliations. 